\title{
Parasitoses intestinais: o que a comunidade sabe sobre este tema?
}

\author{
Parasitic intestinal worm: what the community knows about this theme?
}

\section{Parasitosis intestinales: qué sabe la comunidad sobre este tema?}

Maria Assunta Busato. Universidade Comunitária da Região de Chapecó (UNOCHAPECÓ). Chapecó, SC, Brasil. assunta@unochapeco.edu.br (Autora correspondente)

Daniele Zanoni Dondoni. Universidade Comunitária da Região de Chapecó (UNOCHAPECÓ). Chapecó, SC, Brasil. dani_dondoni@hotmail.com Alécio Leonardo dos Santos Rinaldi. Universidade Comunitária da Região de Chapecó (UNOCHAPECÓ). Chapecó, SC, Brasil.

leorinaldi@unochapeco.edu.br

Lucimare Ferraz. Universidade Comunitária da Região de Chapecó (UNOCHAPECÓ). Chapecó, SC, Brasil. Iferraz@unochapeco.edu.br

\section{Resumo}

As enteroparasitoses constituem um importante problema de saúde pública, contribuindo para as taxas de morbimortalidade devido a doenças infecto-parasitárias em todo o mundo. Objetivo: conhecer as atitudes e percepções da comunidade a respeito de parasitoses intestinais. Métodos: fol aplicado um questionário semiestruturado a 72 pessoas entre 18 e 70 anos para identificar o que elas conheciam sobre parasitoses intestinais. Resultados: a medida de prevenção mais utilizada foi higiene pessoal $(77,7 \%)$, e $66,6 \%$ dos respondentes concordaram que os vermes contaminam o meio-ambiente. Os sintomas mais citados foram dor de barriga e coceira e 72,2\% mencionaram que procuram um médico quando suspeitam de contaminação. Conclusão: os resultados mostram uma forma simplificada de conhecimento, baseada no cotidiano da população, sendo esses achados úteis para o planejamento de futuras campanhas e projetos educacionais.

\section{Abstract}

The enteric parasites are an important public health problem, contributing to the morbimortality rates due to infectious and parasitic diseases world wide. Objective: to understand the attitudes and perceptions of a community about parasitic intestinal worms. Methods: a semi-structured questionnaire was applied to 72 individuals aging from 18 to 70 years old to identify what they knew about intestinal parasites. Results: personal hygiene was the most used preventive measure $(77.7 \%)$ and $66.6 \%$ agreed that the worms contaminate the environment. The most common symptoms mentioned by the participants were abdominal pain and itching. $72.2 \%$ sought for a doctor when they suspected that they were contaminated. Conclusion: the results show that the participants had a simplified form of knowledge based on their everyday life and these findings are useful when planning future campaigns and educational projects.

\section{Resumen}

Las enteroparasitosis constituyen un importante problema de salud pública contribuyendo con las tasas de morbimortalidad por enfermedades infecciosas y parasitarias en todo el mundo. Objetivo: conocer las actitudes y percepciones de la comunidad respecto a las parasitosis intestinales. Métodos: fue realizada una encuesta a 72 personas entre 18 y 70 años para identificar lo que conocían sobre las parasitosis intestinales. Resultados: la prevención más utilizada fue la higiene personal $(77,7 \%$ y $66,6 \%$ creían que los helmintos contaminan el medio ambiente. Los síntomas más citados fueron el dolor de barriga y picazón. 72,2\% buscaban un medico cuando sospechan de contaminación. Conclusión: los resultados muestran una forma simplificada de conocimiento, basado en el cotidiano de la población, siendo estos hallazgos útiles para el planeamiento de futuras campañas y proyectos educacionales.
Palavras-chave:

Enteropatias Parasitárias Meio Ambiente

Saúde Pública

\section{Keywords:}

Intestinal Diseases, Parasitic Environment Public Health

\section{Palabras-clave}

Parasitosis Intestinales Ambiente

Salud Pública 


\section{Introdução}

As parasitoses intestinais constituem um importante problema de saúde pública, pois contribuem para as taxas de morbidade e mortalidade de pessoas em todo o mundo, especialmente em países em desenvolvimento. A transmissão dos parasitos está ligada à condiçôes de vida, como hábitos alimentares, condiçôes de moradia, comportamentos culturais e educacionais, sendo mais prevalentes em populações de baixo nível socioeconômico, não se relacionando diretamente à predisposiçáo racial, genética ou suscetibilidades específicas. ${ }^{1}$ As enfermidades causadas por parasitos são apontadas como fortes indicadores de desenvolvimento socioeconômico de um país. ${ }^{2}$ Estudos realizados nas regiôes sul e sudeste do Brasil encontraram ampla variação na prevalência de parasitoses, entre 23 a 68,9\% e, nos municípios das regióes norte e nordeste, constataram prevalências superiores a $50 \%$. $^{3,4}$

Entre os agravos que as enteroparasitoses podem causar, destacam-se a obstrução intestinal (Ascaris lumbricoides), desnutrição (Ascaris lumbricoides e Trichuris trichiura), anemia ferropriva (ancilostomídeos), diarreia e má absorção (Entamoeba histolytica e Giardia lamblia), sendo proporcional a carga parasitária às manifestaçóes clínicas. ${ }^{5}$

Para que haja intervençóes, é necessário conhecer não apenas o agente etiológico, fatores biológicos e ambientais, mas também o hospedeiro humano, ${ }^{6}$ pois o comportamento humano é fundamental e deve ser levado em conta para o planejamento de pesquisas em doenças parasitárias que incluam fatores culturais, sociais e psicológicos. ${ }^{7}$

O desconhecimento a respeito das medidas preventivas, principalmente entre as populaçóes menos favorecidas, é condicionante para a disseminação das enteroparasitoses. ${ }^{8}$ Desse modo, é fundamental conhecer o comportamento, a percepção, atitudes e conhecimentos das pessoas em relação ao tema, já que as informaçóes obtidas são fundamentais para a elaboração de eventuais ações e programas de intervenção a serem organizados pelos serviços públicos de saúde.

Estudos que visem conhecer o comportamento humano frente a essa problemática são relevantes, uma vez que no Brasil a importância das helmintoses intestinais não comporta contestação, tendo em vista que a distribuição da doença atinge principalmente grupos da população de baixo nível socioeconômico e baixo nível educacional, como já mencionava Vinha, em $1975 .{ }^{9}$

Diante da importância dada ao assunto, realizou-se este estudo com o objetivo de se conhecerem as percepçóes, conhecimento e atitudes de uma população sobre o tema parasitoses. O território escolhido para o desenvolvimento do estudo foi um município do sul do Brasil (27 $5^{\prime} 48^{\prime \prime}$ Sul, $52^{\circ} 37^{\prime} 7^{\prime \prime}$ Oeste), com a parcela da população que apresentava a maior prevalência de parasitoses intestinais do município, segundo estudo realizado em $2009 .{ }^{10}$

\section{Métodos}

Este é um estudo descritivo com abordagem quantitativa, realizado em um município localizado ao oeste do estado de Santa Catarina. De acordo com dados do Atlas Brasil de 2013, ${ }^{11}$ o município possui um IDH de 0.799. Porém, apesar de seu alto índice de desenvolvimento, a mortalidade por doenças infecciosas e parasitárias ainda continua elevada. No ano de 2013, o total foi de 43 óbitos, sendo 18 mulheres e 25 homens.

O instrumento utilizado para a pesquisa foi um questionário adaptado de Mello, ${ }^{7}$ contendo perguntas semiestruturadas, que abordam aspectos sobre o conhecimento da população a respeito de: tipos de parasitoses, ciclo evolutivo de importância epidemiológica (fontes de infecção, habitat do hospedeiro, sobrevida dentro do hospedeiro, vias de eliminação e contaminaçãa do meio-ambiente), sintomas mais comuns e tratamentos utilizados pela população.

O questionário foi aplicado em um Centro Integrado de Saúde que assiste a uma população de aproximadamente 750 famílias, no período de março a agosto de 2011. Foram entrevistadas 72 pessoas, escolhidas de forma aleatória simples, considerando-se na amostra um índice de confiança de 95\%, com uma frequência esperada do fator em estudo de $75 \%$ e pior resultado de $65 \%$. O cálculo amostral foi realizado por meio do programa Epi Info ${ }^{\mathrm{TM}}$, versão 3.5.1. Foram incluídas na pesquisa pessoas com idade entre 18 e 80 anos que se encontravam em espera por atendimento no Centro Integrado de Saúde. Os dados foram analisados utilizando estatística descritiva.

O projeto que deu origem ao estudo foi analisado e aprovado pelo Comitê de Ética em Pesquisa da Universidade Comunitária da Regiâo de Chapecó (UNOCHAPECÓ), número do protocolo ${ }^{\circ} 113 / 10$, atendendo aos requisitos fundamentais da Resolução 196/96 e suas complementares do Conselho Nacional de Saúde/MS. 


\section{Resultados}

Apesar de 97,2\% das pessoas entrevistadas responderem que conhecem ou sabem o que são parasitoses intestinais, menos de $20 \%$ identificaram mais de um tipo de verme ou verminose. Dessas, $85,7 \%$, citaram o A. lumbricoides como um dos conhecidos. Nesse grupo, foram consideradas as indicaçóes de lombriga, áscaris e "branquinho". Outro helminto lembrado por aproximadamente $80 \%$ dos entrevistados foi a Taenia sp. Para esse parasito foram consideradas as indicaçôes de tênia, "pipoquinha" e solitária. A Giardia lamblia, assim como o Schistossoma mansoni e o Necator americanus, somente foram lembrados por $1,4 \%$.

A medida de prevenção mais utilizada pelos entrevistados foi higiene pessoal (77,7\%). Foram consideradas nesse grupo, respostas como higiene, higiene pessoal, lavar as mãos, manter roupas limpas e manter unhas curtas. A segunda forma de prevenção mais lembrada foi o cuidado com água e alimentos. Esse grupo representou $52 \%$ dos entrevistados. A higiene ambiental foi lembrada por menos de $10 \%$ das pessoas.

Quase todos $(97,5 \%)$ os participantes da pesquisa citaram que é possível se contaminar com algum tipo de parasito ingerindo alimentos mal lavados ou colocando a mão suja na boca. A maioria também concordou que nadar em água parada é fator de risco para contaminação. Além disso, 87,5\% dos participantes indicaram que é possível se contaminar ingerindo carne mal passada ou andando descalço. Mais da metade dos participantes afirmaram que é possível ocorrer transmissão por meio do espirro de pessoas contaminadas $(65,2 \%)$ ou de relação sexual $(62,5 \%)$ com parceiro contaminado com algum tipo de verme.

Sobre a alocação dos parasitos intestinais no organismo, 68\% afirmaram que esses se instalam no intestino, no estômago e no sangue ( $18 \%$ e $11,1 \%$, respectivamente). Outros $(4,16 \%)$ mencionaram que os parasitas podem se alojar nos pulmôes, no cérebro ou em todos os órgãos. Contudo, cerca de $20 \%$ não sabiam onde os vermes se abrigam no organismo após a contaminação.

A maioria (68\%) dos participantes afirmou que os vermes são eliminados do organismo por meio das fezes, e aproximadamente um quarto dos entrevistados não soube de que maneira os parasitos saem do corpo. Outras respostas, tais como eliminação por vômito, urina ou espirro somaram $15 \%$ das afirmaçóes.

Os respondentes $(66,6 \%)$ concordaram que os vermes contaminam o meio-ambiente ao saírem do organismo, sendo que $41,6 \%$ e 39,5\% indicaram a água e a terra, respectivamente, como principal local a ser contaminado, e um terço indicou os alimentos como alvos da contaminaçáo. Mais de 35\% não souberam responder a respeito do que poderia ser contaminado.

Entre os sinais e sintomas gerados pelas parasitoses intestinais, os mais citados foram dor de barriga e coceira (30\%). Das pessoas que fizeram parte deste estudo, 75\% citaram outros sintomas como dor de cabeça, mal-estar, falta de ar, alergia, dor no corpo, sangue nas fezes, irritação, agitação, diarreia, feridas, anemia, tosse, emagrecimento, insônia, vômito, palidez, vontade de comer doces e aumento do apetite. Por outro lado, a maioria $(72,2 \%)$ indicou que a conduta ideal a ser tomada em caso de contaminação com enteroparasitos é a de procurar um médico para que esse confirme o diagnóstico e receite o tratamento farmacológico correto. Mais de um quarto da população pesquisada acreditava que se deve tomar antibiótico em caso de contaminação. Aproximadamente metade dessa população utilizava o chá como recurso terapêutico e $18 \%$ acreditavam que curandeiros ou benzedeiras podem ser resolutivos no tratamento das verminoses.

\section{Discussão}

Neste estudo, foram analisados alguns aspectos sobre conhecimentos, atitudes e percepção de uma comunidade atendida por um Centro Integrado de Saúde em relação às parasitoses intestinais. Os resultados obtidos mostram que a população tem uma codificação própria para nominar os helmintos intestinais, a saber: bicha, lombriga, áscaris, solitária, 'branquinho' para se referirem ao Ascaris lumbricoides, 'pipoquinha' para a Taenia sp., entre outros.

Um estudo similar desenvolvido por $\mathrm{Mello}^{7}$ sobre conhecimentos, atitudes e percepção da população em relação aos helmintos intestinais também mostrou que a população apresenta uma forma própria para nominar os helmintos intestinais: bicha, lombriga, bichinho miúdo, geográfico, solitária, e outros. 
A população estudada citou que o contágio se dá pela água e alimentos contaminados, pela falta de higiene corporal, nadar em água parada, etc. Porém, algumas respostas demonstraram um conhecimento restrito sobre o assunto, entre elas, a transmissão dos parasitos por meio do espirro, dos fluídos sexuais, pelo uso de roupas de uma pessoa contaminada ou de se encostar em alguém com parasitose intestinal. Isso evidencia a necessidade de realização de açôes junto à comunidade, que possam orientar a população sobre os cuidados e a prevençáo dessas enfermidades observando, em especial, o ambiente em que vive a população, suas condiçóes de trabalho e o acesso aos serviços de saúde. No pensamento de Fleury-Teixeira, ${ }^{12}$ as condiçôes de habitação e ambientais do peridomicílio, a existência de restrições no acesso à alimentação e a outros bens fundamentais, as características físicas das atividades realizadas no trabalho, assim como as condiçóes do ambiente em que se realiza o trabalho podem implicar uma série de riscos à saúde que, em geral, estáo além da possibilidade de controle por parte dos indivíduos.

$\mathrm{Na}$ mesma direção, um estudo realizado em Belo Horizonte ${ }^{13} \mathrm{com}$ alunos e professores sobre conhecimento, opiniōes e prevalência de helmintíase, verificou que entre os alunos de $1^{\mathrm{a}}$ a $4^{\mathrm{a}}$ séries, $88,3 \%$ sabiam que não se deve colocar as mãos sujas na boca para não se infectarem. Um grande número de crianças $(77,2 \%)$ considerava possível se infectar por vermes usando o mesmo copo de alguém que esteja contaminado. Andar descalço foi para $84,6 \%$ das crianças, fator relevante de contaminação. Também, um estudo ${ }^{14}$ que envolveu estudantes de $6^{a}$ série de duas escolas de $1^{\mathrm{o}}$ grau do município de Serra Talhada (PE), para avaliar o conhecimento sobre as parasitoses intestinais, verificou que em relação a pergunta "como a pessoa se contamina pelos vermes?” $50 \%$ dos alunos indicaram o hábito de andar descalço como a principal forma de contaminação pelas verminoses.

É importante considerar que as visitas ao bairro ou à escola para conhecer os hábitos e percepçốes da população sobre uma temática não dão conta de apreender o que aflige as pessoas, o que mais afeta sua saúde naquele espaço e ambiente. ${ }^{15}$ Portanto, as respostas dadas aos questionamentos durante o diálogo mantido com as pessoas dessa comunidade podem ser pontuais e representar a percepção daquele momento em particular. Ainda assim, considera-se o que as pessoas pontuam sobre a temática como, por exemplo, os lugares a serem contaminados com a eliminação dos parasitos que são a água, a terra e os alimentos, porém não relacionam a eliminação dos parasitos à contaminação ambiental ou ainda à contaminação fecal-solo-doença.

Neste estudo, pode-se perceber que a população tinha conhecimento sobre sinais e sintomas gerados pelas parasitoses, mas recorrendo ao que seus pais e avós diziam, pois ressalta-se que essas informaçôes são oriundas de um conhecimento popular transmitido ao longo de geraçóes. Entre os sinais e sintomas das parasitoses foram mencionados dor de barriga, coceira, falta de apetite, fraqueza, vontade de comer doces, irritação, diarreia, anemia, emagrecimento, alteraçóes no sono, entre outros. Observa-se que o conhecimento empírico, ou de vivência, não dista muito do que a literatura aponta. Esses sintomas também foram lembrados pela população de Sáo Carlos (SP), no estudo de Mello7que mostrou habilidades semelhantes das pessoas em reconhecer as categorias de sinais e sintomas da doença causada por enteroparasitos.

Quando essa população identifica os sintomas de parasitoses, embora a conduta a ser tomada seja a de procurar um médico, somente $72,2 \%$ procuram esse tipo de ajuda, sendo que o restante faz automedicação, uso de chás e recorrem a curandeiros ou benzedeiras. O estudo de Mello realizado em Chapecó ${ }^{7}$ também identificou que a população que utilizava um serviços de saúde valorizava o uso de chás caseiros e simpatias no tratamento das crianças com vermes, assim como consulta a benzedeiras.

Nesse sentido, na perspectiva do pensamento do educador Paulo Freire ${ }^{16}$ segundo o qual "não há saber mais, nem saber menos, há saberes diferentes", é compreensível que as pessoas busquem os tratamentos caseiros com chás e as benzedeiras para a cura dos vermes, pois o conhecimento popular está sendo acionado no combate aos males que afetam suas famílias.

Assim, pode-se perceber uma grande força do conhecimento religioso e empírico fazendo parte do cotidiano dos entrevistados. O conhecimento religioso representa aquelas verdades a que os homens chegaram não com o auxílio puro e simples da inteligência, mas mediante a aceitação dos dados da revelação divina e da fé. ${ }^{17}$ Já o empirismo, segundo Freire, é o conhecimento do povo, obtido ao acaso, a partir da observação dos acontecimentos e das relaçôes do mundo material exterior, por meio da qual o homem toma consciência das experiências alheias, incorporando, principalmente pela tradição, o legado das ideias transmitidas de geração a geração.

A partir da observação dos dados obtidos, identificou-se também uma relação direta entre baixo grau de escolaridade, baixo nível socioeconômico e respostas insatisfatórias, caracterizando pouco conhecimento dos respondentes sobre as parasitoses. Apesar de haver poucas referências na literatura relacionando grau de escolaridade e conhecimento sobre parasitos intestinais, este estudo evidenciou que entre os entrevistados que disseram não conhecer o que são vermes, um está incluído no grupo 
dos analfabetos e o outro cursou até a quarta série do ensino fundamental. As pessoas que apresentaram as respostas mais satisfatórias concluíram o ensino médio (31,95\%), já os que cursaram somente até a quarta série, ensino fundamental ou analfabeto $(68,05 \%)$ mostraram um conhecimento muito simplificado, baseado apenas no cotidiano. Considerando que mais de metade da população possuía até o ensino fundamental completo e, destes, a maioria tinha estudado até a $4^{a}$ série primária, é compreensível que o conhecimento dessa população seja insuficiente para que perceba e coloque em prática açôes de prevenção às parasitoses.

Entendendo que há necessidade de melhorar o acesso à educação, torna-se imprescindível que essa temática seja trabalhada nas escolas e com grupos de moradores. Os serviços públicos de saúde também deveriam atentar para a necessidade de orientaçóes sobre essa problemática para a população que busca atendimento. É importante destacar a relevância do trabalho na atenção primária à saúde, ${ }^{18}$ mais especificamente no contexto da estratégia saúde da família, que deve partir de uma base comunitária de forma dialógica e participativa. Por outro lado, como evidenciam Rodrigues e Ribeiro, ${ }^{19}$ os trabalhadores dos serviços de saúde devem atentar para não 'depositar' o conhecimento nos usuários, considerando que eles "não sabem nada".

Para exemplificar a importância da educação em saúde na prevenção e na redução da incidência de contaminados, pode-se citar a experiência realizada em Estiva Gerbi (SP), ${ }^{20}$ no período de 2000 a 2002, com pais e escolares de 0 a 7 anos de idade, em que foi realizada uma oficina de trabalho no sentido de informar e discutir parasitos intestinais a fim de complementar o tratamento médico. Após dois anos, em uma nova avaliação, a prevalência de enteroparasitoses, que era de 23,5\%, diminuiu para 6,6\% entre os alunos que participaram da oficina de trabalho. Porém, na escola como um todo, devido ao ingresso de novos estudantes que não tiveram contato com a oficina, a prevalência era ainda de 23,3\%, o que reitera a importância de uma educação continuada no controle das enteroparasitoses. No entanto, é preciso que informaçôes repassadas vejam contextualizadas com a realidade em que vive a população ${ }^{21}$.

Embora a informação, no que condiz à educação em países em desenvolvimento, ainda seja um privilégio para poucos, a primeira Conferência Internacional sobre Promoção da Saúde, realizada em Ottawa, Canadá, em novembro de 1986, deixa claro em sua carta de intençôes que a promoção da saúde trabalha por meio de ações comunitárias concretas e efetivas no desenvolvimento das prioridades, na tomada de decisão, na definição de estratégias e na sua implementação, visando à melhoria das condiçóes de saúde. ${ }^{22} \mathrm{O}$ centro desse processo é o incremento do poder das comunidades, a posse e o controle dos seus próprios esforços e destinos. É importante aproveitar os recursos humanos e materiais das próprias comunidades para intensificar a ajuda e o apoio social e desenvolver a participaçáo popular nos assuntos de saúde. Isso requer um total e contínuo acesso à informação, às oportunidades de aprendizado para os assuntos de saúde, assim como apoio financeiro adequado.

\section{Conclusão}

Este estudo evidencia que a populaçáo estudada possuía lacunas de conhecimento a respeito das parasitoses intestinais. Assim sendo, é importante realizar açôes sócio educacionais com a população no intuito de informá-la sobre os tipos de parasitoses intestinais, medidas preventivas, ciclo de contaminação e eliminação, sintomas e tratamentos corretos para a eliminação desses parasitos, uma vez que os mesmos são pouco conhecidos pela comunidade.

É necessário atuar com medidas que estimulem mudanças de comportamento em relação ao autocuidado, prevenção e profilaxia das parasitoses, no intuito de melhorar a qualidade de vida da população e minimizar a contaminação por parasitos. Para tanto, faz-se necessário o desenvolvimento de atividades de educação em saúde pelos profissionais responsáveis pela comunidade da área de abrangência da Unidade de Saúde. Além disso, este tema deveria ser abordado e trabalhado nas escolas e grupos comunitários para que as pessoas, famílias e comunidades busquem meios de enfrentar as vulnerabilidades presentes no ambiente, em relaçáo à contaminação parasitária.

\section{Referências}

1. Duncan BB, Schmidt MI, Giuliani ERJ. Medicina ambulatorial: condutas de atenção primária baseada em evidências. $3^{\mathrm{a}}$ ed. Porto Alegre: Artmed; 2004.

2. Moraes RG, Goulart EG, Leite IC. Parasitologia e micologia humana. $4^{\text {a }}$ ed. Rio de Janeiro: Cultura Médica; 2000.

3. Santos JF, Correia JE, Gomes SSBS, Silva PC, Borges FAC. Estudo das parasitoses intestinais na comunidade carente dos bairros periféricos do município de Feira de Santana (BA), 1993-1997. Sitientibus. 1999;20:55-67. http://www2.uefs.br/sitientibus/pdf/20/estudo_das_parasitoses.pdf. 
4. Alves Jr, Macedo HW, Ramos Jr AN, Ferreira LF, Gonçalves MLC, Araújo A. Parasitoses intestinais em região semi-árida do Nordeste do Brasil: resultados preliminares distintos das prevalências esperadas. Cad Saúde Pública. 2003;19(2):67-670. http://dx.doi.org/10.1590/S0102-311X2003000200034

5. Ferreira UM, Ferreira CS, Monteiro CA. Tendência secular das parasitoses intestinais na infância na cidade de São Paulo (1984-1996). Rev Saúde Pública. 2000;34(6 Supl):73-82. http://dx.doi.org/10.1590/S0034-89102000000700010

6. Pedrazzani ES, Mello DA, Pizzigatti CP, Barbosa CAA. Aspectos educacionais da intervenção em helmintoses intestinais, no subdistrito de Santa Eudóxia, Município de São Carlos - SP. Cad Saúde Pública. 1990;6(1):74-75. http://dx.doi.org/10.1590/S0102-311X1990000100008

7. Mello DA, Pripas S, Fucci M, Santoro MC, Pedrazzani ES. Helmintoses intestinais: I. conhecimentos, atitudes e percepção da população. Rev Saúde Pública. 1988;22(2):140-149. http://dx.doi.org/10.1590/S0034-89101988000200010

8. Munhoz RAR, Faintuch MB, Valtorta A. Enteroparasitoses em pessoal de nutrição de um hospital geral: incidência e valor da repetição dos exames. Rev HospClinFac Med S Paulo. 1990;45(2):57-60.

9. Vinha C. Necessidade de uma política sanitária nacional para o combate às parasitoses intestinais. Rev Soc Bras Med Trop. 1975;10:297-301. http://dx.doi.org/10.1590/S0037-86821976000500005

10. Poli G, Tonini P, Busato MA. Prevalência das parasitoses intestinais no município de Chapecó. Resumo apresentado no Seminário Integrado Ciência Brasil. Unochapecó, Chapecó-SC;2009.

11. Brasil. Atlas de Desenvolvimento Humano no Brasil 2013 [Online]. [acesso em 2013 Jul 29]. Disponível em: http://www.atlasbrasil.org.br/2013/perfil/chapeco_sc.

12. Fleury-Teixeira P. Uma introdução conceitual à determinação social da saúde.Saúde Debate. 2009;33(83):380-387. http://www.cebes.org.br/media/file/SDv33n83.pdf.

13. Santos MG, Moreira MM, Malaquias MLG, Schall VT. Educação em saúde em escolas pública de $1^{\circ}$ grau da periferia de Belo Horizonte, MG, Brasil: II. Conhecimentos, opiniões e prevalência de helmintísase entre alunos e professores. Rev Inst Med Trop. 1993;35(6):573-579. http://dx.doi.org/10.1590/S0036-46651993000600015

14. Barbosa RN, Costa MMR, Lima AMS, Cavalcante ECC, Santos EM. Conhecimento de escolares frente às parasitoses intestinais. Serra Talhada, PE, 2009. [acesso em 2014 Dez 20]. Disponível em: http://www.eventosufrpe.com.br/jepex2009/cd/resumos/R0463-1.pdf.

15. Torrezan RM, Guimarães RB, Furlanetti MPFR. A importância da problematização na construção do conhecimento em Saúde Comunitária. Trab Educ Saúde. 2012;10(1):107-124. http://dx.doi.org/10.1590/S1981-77462012000100007

16. Freire P. Pedagogia do oprimido.42ª ed. São Paulo: Paz e Terra; 2005.

17. Braga WFL. O conhecimento.Faculdade de Direito de Campos. [acesso em 2014 Dez 20]. Disponível em: http://www.fdc.br/Artigos/.. \Arquivos\Artigos $\backslash 14 \backslash 0$ Conhecimento.pdf.

18. Aguiar LFP, Lima EM, Azevedo LA, Lira MG, Martildes MJR, Moreira AEMM, et al. Compreendendo a comunidade: experiência da equipe interdisciplinar do Bom Jardim - Fortaleza (CE). Rev Bras Med Fam Comunidade. 2008[acesso em 2014 Dez 20];4(14):140-145. Disponível em: http://www.rbmfc.org.br/rbmfc/article/view/197.

19. Rodrigues CC, Ribeiro KSQS. Promoção da saúde: a concepção dos profissionais de uma unidade de saúde da família. TrabEduc Saúde. 2012;10(2):235-255. http://dx.doi.org/10.1590/S1981-77462012000200004

20. Ferreira GR, Andrade CFS. Alguns aspectos socioeconômicos relacionados a parasitoses intestinais e avaliação de uma intervenção educativa em escolares de Estiva Gerbi, SP.RevSocBras Med Trop. 2005;38(5):402-405. http://dx.doi.org/10.1590/S0037-86822005000500008

21. Freitas JD, Porto MF. Por uma epistemologia emancipatória da promoção da saúde. Trab Educ Saúde. 2011;9(2):179-200. http://dx.doi.org/10.1590/S1981-77462011000200002 